\title{
Long-term outcome following bilateral lateral rectus recession for intermittent exotropia
}

\author{
Jutima Patlidanon ${ }^{\mathrm{a}}$, Parnchat Pukrushpan ${ }^{\mathrm{a}}$, Supharat Jariyakosol ${ }^{\mathrm{a}}$, Pokpong Praneeprachachon ${ }^{\mathrm{a}, \mathrm{b}}$ \\ ${ }^{a}$ Department of Ophthalmology, Faculty of Medicine, Chulalongkorn University, Bangkok 10330, \\ ${ }^{b}$ Rutnin Eye Hospital, Bangkok 10110, Thailand
}

\begin{abstract}
Background: Bilateral lateral rectus recession is the most common surgical treatment of intermittent exotropia in children. Success rates in previous studies differ because longer follow-up periods may change success rate as the eyes drift out with time. Initial overcorrection was thought to be appropriate management, but the preferred initial postoperative deviation has not been well quantified.

Objective: To study long-term outcomes ( $\geq 3$ years) of bilateral lateral rectus recession for intermittent exotropia and to evaluate factors influencing long-term success.

Methods: This retrospective descriptive study reviews patients with intermittent exotropia who underwent bilateral lateral rectus recession with a minimum follow up of 3 years. Long-term motor outcome was classified as good (within 8 prism diopters (PD) of esodeviation and 10 PD of exodeviation), acceptable (exodeviation 11-20 PD), and poor (esotropia >8 PD or exotropia >20 PD). Stereoacuity testing pre- and postoperatively was used to evaluate sensory outcome. Correlation between long-term outcome and various pre- and postoperative factors were analyzed.

Results: A total of 59 patients met the selective inclusion criteria. The mean age at time of surgery was 7.1 years with a mean follow-up time of 4.5 years. At the time of the last visit, 69\% (41 patients) had good outcomes, 24\% (14 patients) had acceptable outcomes, and 7\% (4 patients) had poor outcomes. Initial postoperative ocular deviation significantly correlated with long-term outcome $(P<0.05)$. Mean initial postoperative ocular deviation of patients with good outcome was esodeviation 12.7 PD (range ortho to esodeviation 25 PD). In 45 patients whose stereoacuity could be assessed, 58\% (26 patients) had stereoacuity improvement.

Conclusion: Good long-term motor outcome can be achieved in 69\% of patients following bilateral lateral rectus recession for intermittent exotropia. Initial postoperative ocular deviation correlated with long-term motor success.
\end{abstract}

Keywords: Bilateral lateral rectus recession, intermittent exotropia, strabismus

Intermittent exotropia is the most common type of divergence strabismus in young children, and has high prevalence in countries in tropical regions such as Thailand. Without treatment, intermittent exotropia usually progresses by increasing in angle of deviation, worsening control, decreased stereoacuity, and if left untreated, results in constant exotropia.

The standard surgical procedure is bilateral lateral rectus recession [1]. Success rates of this procedure are not consistent in previous studies (42.2\%-63\%) [2-9]. Exotropic drift is believed to be the reason of unsuccessful outcome. However, a majority of patients were found to have developed postoperative

Correspondence to: Parnchat Pukrushpan, MD, Department of Ophthalmology, Faculty of Medicine, Chulalongkorn University, Bangkok 10330, Thailand. E-mail: parnchat@gmail.com exotropic drift with time. Therefore, initial overcorrection is thought to be appropriate management, but the preferred initial postoperative deviation has not been well quantified [10]. The purpose of this study is to examine the long-term outcome (at least 3 years) of bilateral lateral rectus recession for patients with intermittent exotropia and to determine the initial postoperative angle deviation that best correlates with long-term success.

\section{Materials and methods}

This study was approved by the King Chulalongkorn Memorial Hospital and Rutnin Eye Hospital Institutional Review Board. It is a retrospective descriptive analytical study that reviewed patients with intermittent exotropia who underwent bilateral lateral recession during September 
2005 to May 2008 at King Chulalongkorn Memorial Hospital or Rutnin Eye Hospital using the same surgical technique by 2 surgeons (Praneeprachachon P. and Pukrushpan P.).

Patients with intermittent exotropia who, underwent BLR recession as initial surgery with postoperative follow-up time of at least 3 years, were included. Those with coexisting vertical deviation, oblique muscle dysfunction, significant A or V pattern, restrictive or paralytic strabismus, craniofacial malformations, prior strabismus surgery or botulinum toxin injection, reduced visual acuity (except refractive error or mild amblyopia that defined as best corrected visual acuity better than 20/40), and significant neurological impairment were excluded.

Collected demographic data included: age at time of surgery, sex, and any underlying diseases. Clinical data included; best corrected visual acuity, ocular deviation at distance $(20 \mathrm{ft} ; 6.1 \mathrm{~m})$ and near $(1 \mathrm{ft}$; $0.3 \mathrm{~m}$ ) at preoperative, postoperative at 1 week and final visit. Near stereoacuity was tested using pre and postoperative Titmus stereo tests. Long-term motor outcome was classified according to potential of binocular fusion as good outcome (within $8 \mathrm{PD}$ of esodeviation and $10 \mathrm{PD}$ of exodeviation), acceptable outcome (exodeviation 11-20 PD), and poor outcome (esodeviation $>8$ PD or exodeviation $>20$ PD). Sensory outcome was classified as good (stereoacuity $\leq 60$ seconds of arc), poor (>60 seconds of arc), and no stereoacuity. Secondary operation because of undesirable outcome were recorded.

Long-term motor outcome and sensory outcome was evaluated by proportion analysis. The correlation between preoperative deviation, 1 week postoperative deviation, age at surgery, and long-term motor outcome were analyzed by univariate and multivariate analysis.
Correlation between sex and long-term motor outcome were analyzed using an unpaired $t$ test.

\section{Results \\ Pre-operative characteristics}

A total of 59 patients met the inclusion criteria; 26 male (44\%), 33 female (56\%), with a mean age at surgery 7.1 years (range 2.3-15). Mean follow up time was 4.5 years (range 3-7). Mean initial preoperative exodeviation was 27 prism diopters, PD (range 1845 ) at distance, and 29 PD (range 18-50) near. Preoperative best corrected visual acuity in the worse eye was: 20/20 34\% (20 patients), 20/25 20\% (12 patients), 20/30 25\% (15 patients), 20/40 17\% (10 patients), and CSM 3\% (2 patients).

\section{Motor outcome}

At last follow up visit, 69\% (41 patients) had good motor outcome, 24\% (14 patients) had acceptable outcome, and 7\% (4 patients) had poor outcome (Table 1). Two patients, who had poor outcome, underwent reoperation for consecutive esotropia. An average first week postoperative ocular deviation at distance is shown in Table 1. An average of the first week postoperative deviation in patients who achieve good long-term outcome was esodeviation 12.7 PD (range from orthotopia to esodeviation $25 \mathrm{PD}$ ) (mode and median were $12 \mathrm{PD}$ ). During the follow up period, $83 \%$ of patients had exotropic drift with average exotropic drift of 15.7 PD. Percentage of patients with exotropic drift was $85 \%$ in good, $71 \%$ in acceptable, and $50 \%$ in poor motor outcome groups. Average exotropic drift was 15.1 PD in good, 12.8 PD in acceptable, and 47.0 PD in poor motor outcome groups.

Table1. Preoperative and initial postoperative data classified according to long-term success

\begin{tabular}{|c|c|c|c|}
\hline & $\begin{array}{l}\text { Good outcome } \\
\text { (XT } 10 \text { PD - ET } 8 \text { PD) }\end{array}$ & $\begin{array}{l}\text { Acceptable outcome } \\
\text { (XT 11 - } 20 \text { PD) }\end{array}$ & $\begin{array}{l}\text { Poor outcome } \\
\text { (ET }>8 \text { PD or XT }>20 \text { PD) }\end{array}$ \\
\hline No. of patients & $41(69 \%)$ & $14(24 \%)$ & $4(7 \%)$ \\
\hline Age, years (range) & $6.4(2.4-12)$ & $7.6(2.25-15)$ & $6(3-10)$ \\
\hline Sex: male, female & 16,25 & 8,6 & 3,1 \\
\hline Preop deviation at distance, PD (range) & XT 26.6(18-45) & XT 29.5(18-40) & XT 26.3(20-30) \\
\hline Preop deviation at near, PD (range) & XT’27.5(18-50) & XT’31.4(20-45) & XT’35 \\
\hline $\begin{array}{l}\text { First week postoperative deviation at } \\
\text { distance, PD (range) }\end{array}$ & $\begin{array}{l}\text { ET } 12.7 \\
\text { (Ortho-ET 25) }\end{array}$ & $\begin{array}{l}\text { ET 8.1 } \\
\text { (XT 8-ET 20) }\end{array}$ & $\begin{array}{l}\text { ET 26 } \\
\text { (ET 4-40) }\end{array}$ \\
\hline $\begin{array}{l}\text { First week postoperative deviation at near, } \\
\text { PD (range) }\end{array}$ & $\begin{array}{l}\text { ET } 8.8 \\
\text { (Ortho-ET’25) }\end{array}$ & $\begin{array}{l}\text { ET } 8.0 \\
\text { (XT’8-ET’30) }\end{array}$ & $\begin{array}{l}\text { ET 19 } \\
\text { (ET’6-30) }\end{array}$ \\
\hline
\end{tabular}

$\mathrm{XT}=$ exodeviation, ET = esodeviation, Ortho = orthotopia 


\section{Sensory outcome}

Of 59 preoperative patients, $41 \%$ (24 patients) had good stereoacuity, 41\% (24 patients) had poor stereoacuity, 3\% (2 patients) had no stereoacuity, and $15 \%$ (9 patients) were unable to perform stereotest. Postoperatively, out of a total of 57 patients, 68\% (39 patients) had good stereoacuity, 25\% (14 patients) had poor stereoacuity, and 7\% (4 patients) were unable to perform stereotesting. Two patients were excluded from postoperative data collection because of reoperation.

Preoperative and postoperative data were obtained from 44 patients. Comparative stereoacuity outcome was classified according to long-term motor success as shown in Table 2.

\section{Comparison analysis}

Two patients were excluded from comparison analysis because of reoperation. According to univariate analysis, there was no significant correlation between long-term outcome and preoperative ocular deviation $(P=0.24)$, or age at surgery $(P=0.07)$. There was also no significant difference in long-term outcome between male and female patients $(P=0.59)$ by independent sample $t$ test.

First week postoperative ocular deviation was the only factor that significantly correlated with long-term postoperative outcome by univariate analysis $(P=0.002, \mathrm{R} 0.402)$ and multivariate analysis $\left(P=0.002, R^{2} 0.164\right)$.

The predicted long-term postoperative distance deviation equation from linear regression was “ $y=-9.36+0.38 x ; R^{2}=0.162 ”(y=$ long-term postoperative distance deviation, $x=$ the first week postoperative distance deviation).

\section{Discussion}

Success rates of bilateral lateral rectus recession for intermittent exotropia differ in previous studies (42.2\%-63\%). There are many explanations that could account for this. One being that a longer followup period may change the success rate as the eye drifts out (exotropic drift) with time. In this study, we reported long-term outcomes with a minimum follow up period of 3 years, which is longer than that in previous studies [2, 3, 5-9, 11, 12]. The success rate of bilateral lateral rectus recession for intermittent exotropia in this study was $69 \%$, which is not inferior to others, but success rates may be influenced by different definitions of success and follow up periods in each study.

It was suggested by previous studies that initial undercorrection may lead to poor long-term outcome [13] and initial overcorrection is important to achieve good long-term outcome [2]. However, there is no exact or "magic" number of the preferred initial ocular deviation for desirable long-term outcomes. Moreover, according to our study, the most common cause of reoperation was consecutive esodeviation. Leow and colleagues found that initial overcorrection is not necessary because they found that initial postoperative deviation did not significantly affect long-term outcome [3]. In this study, we also evaluated correlations between initial postoperative deviation and long-term outcome.

We used a postoperative period of 1 week to avoid effects from shorter postoperative times that would otherwise influence the measurement; such as chemosis and postoperative pain. We found that initial postoperative deviation is the only factor that correlated with long-term outcome $(P<0.05)$, but this correlation was not strong $(R=0.04)$.

We found that $83.0 \%$ of the patients developed exotropic drift. Average exotropic drift was 15.7 PD and thus we also believe that initial postoperative overcorrection provides a higher chance to achieve good long-term outcome. In the current study, the average initial postoperative distance deviation in patients with good long-term outcome was esodeviation 12.7 PD (range from orthotopia to esodeviation

Table 2. Change of stereoacuity following bilateral lateral rectus recession, classified according to long-term motor outcome

\begin{tabular}{llll}
\hline Long-term motor outcome & \multicolumn{2}{c}{ Change in stereoacuity (no. of patients) } \\
\cline { 2 - 4 } & Improved & Worsen & Stable \\
\hline Good motor outcome & 20 & 2 & 10 \\
Acceptable motor outcome & 5 & 1 & 5 \\
Poor motor outcome & 1 & - & - \\
Total & $26(59 \%)$ & $3(7 \%)$ & $15(34 \%)$ \\
\hline
\end{tabular}


$25 \mathrm{PD}$ ), which is comparable to previous studies that recommend initial overcorrection of 0-20 PD [2, 12, 14-16].

We used only ocular deviation at distance to evaluate the outcome. This was because distance deviation is usually worse than near deviation according to the nature of this disease. However, if using near deviation to evaluate outcome, some good outcome patients may fall into acceptable or poor outcome groups.

Unlike our study, previous studies rarely included stereopsis evaluation. Here, we aimed to examine sensory outcome, which provided findings that $45.6 \%$ of patients had improved stereoacuity that was comparable with the findings of Ekdawi and colleagues [4]. Often used in strabismus surgical studies, retrospective study designs such as this have limitations because of incomplete data collection and less power for comparison of results. Thus, a prospective study may help minimize these limitations in the future.

\section{Conclusions}

Good long-term motor outcome can be achieved in $69 \%$ of patients following bilateral lateral rectus recession for intermittent exotropia. Initial postoperative ocular deviation correlated with longterm motor success.

\section{Acknowledgements}

This study was supported by the Ratchadaphiseksomphot Fund. This material has been presented at the $29^{\text {th }}$ Annual Academic Meeting of the Royal College of Ophthalmologists of Thailand on May 24, 2012. The authors declare no conflicts of interest to report.

\section{References}

1. Burien HM. Exodeviations: their classifications, diagnosis and treatment. Am J Ophthalmol. 1966; 62: 1161-6.

2. Ruttum MS. Initial versus subsequent postoperative motor alignment in intermittent exotropia. J AAPOS. 1997;1:88-91.

3. Leow PL, Ko ST, Wu PK, Chan CW. Exotropic drift and ocular alignment after surgical correction for intermittent exotropia. J Pediatr Ophthalmol Strabismus. 2010; 47:12-6.

4. Ekdawi NS, Nusz KJ, Diehl NN, Mohney BG. Postoperative outcomes in children with intermittent exotropia from a population-based cohort. J AAPOS. 2009; 13:4-7.

5. Lee SY, Lee YC. Relationship between motor alignment at postoperative day 1 and at year 1 after symmetric and asymmetric surgery in intermittent exotropia. Jpn J Ophthalmol. 2001; 45:167-71.

6. Richard JM, Parks MM. Intermittent exotropia: surgical results in different age groups. Ophthalmology. 1983; 90:1172-7.

7. Chia A, Seenyen L, Long QB. Surgical experiences with two-muscle surgery for the treatment of intermittent exotropia. JAAPOS. 2006; 10:206-11.

8. Ing MR, Nishimura J, Okino L. Outcome study of bilateral lateral rectus recession for intermittent exotropia in children. Tr Am Ophth Soc. 1997; 95: 433-52.

9. Scott E. Olitsky, MD. Early and late postoperative alignment following unilateral lateral rectus recession for intermittent exotropia. J Pediatr Ophthalmol Strabismus. 1998; 35:146-8.

10. Von Noorden GK. Binocular vision and ocular motility. St. Louis: Mosby. 1980; 323.

11. Stoller SH, Simon JW, Lininger LL. Bilateral lateral rectus recession for exotropia: a survival analysis. J Pediatr Ophthalmol Strabismus. 1994; 31:89-92.

12. Scott WE, Keech R, Mash AJ. The postoperative results and stability of exodeviations. Arch Ophthalmol. 1981; 99:1814-8.

13. Pineles SL, Ela-Dalman N, Zvansky AG, Yu F, Rosenbaum AL. Long-term results of the surgical management of intermittent exotropia. Journal of AAPOS. 2010; 14:298-304

14. Raab EL, Parks MM. Recession of the lateral recti: early and late postoperative alignments. Arch Ophthalmol. 1969; 82:203-8.

15. McNeer KW. Observations on the surgical overcorrection of childhood intermittent exotropia. Am Orthoptic J. 1987; 37:135-50.

16. Souza-Dias C, Uesugui CF. Postoperative evolution of the planned initial overcorrection in intermittent exotropia: 61 cases. Binocular Vis Eye Muscle Surg Q. 1993; 8:141-8. 